\title{
Visual Evidence at the Nanoscale
}

\author{
Otávio Bueno*

\section{Introduction}

Research at the nanoscale $\left(10^{-7}\right.$ to $10^{-9}$ meters) raises a number of intriguing philosophical issues. In this paper, I address one of them: the role of what can be called "visual evidence" in the construction and assessment of nanophenomena.

First, a clarification is in order regarding the concepts of visual evidence and nanophenomena. It might be thought that the former expresses a redundancy whereas the latter is an oxymoron. After all, at least if we follow its Latin etymology, evidence emerges from what is obvious to the eye (and thus can be seen). In this sense, any evidence should then be visual. However, once the concept of evidence is formulated in the context of certain philosophical views, this immediate link to a visual experience need not be maintained - although, ultimately, there will always be such a link. Having said that, breaking the link with the observable is precisely what happens in the case of some of the most influential models of evidence. Rather than keeping a close link to what can be visually perceived, these models stress the way in which evidence supports certain theories - in particular, by making more likely that such theories be true.

With regard to "nanophenomena", it may be argued that the word "phenomena", at least etymologically, stands for what appears, what can be seen. And if we restrict what can be seen to what can be seen without the use of instruments (such as various kinds of microscopes), then simply nothing at the nanoscale could be literally seen. Nanophenomena turn out to be an impossibility. However, once again, if phenomena are understood in the context of certain philosophical conceptions, they need not be tied directly only to what literally appears to our unaided eyes. Phenomena may stand for a certain cluster of events that are stable and regular enough to require some kind of explanation by our theories. Clearly, phenomena will involve something that can be seen: the items with respect to which our theories will be taken to be empirically adequate or not. But this doesn't mean that the phenomena themselves are literally observable (without any use of instruments). Of course, if one invokes a broader conception of observable - according to which items detected by means of microscopes can be said to be observable - then talk of nanophenomena is, again, perfectly fine.

* Otávio Bueno is Professor of Philosophy at the University of Miami. He works in philosophy of science, philosophy of mathematics and philosophy of logic, and is currently writing a book on the epistemology of microscopy. Visual evidence plays an important role in this project.

Spontaneous Generations 2:1 (2008). ISSN 1913-0465. University of Toronto 
In what follows, I will offer a formulation of visual evidence, and indicate how the concept can be used to make sense of some of the work that is currently done at the nanoscale. As an illustration, I will focus on the so-called DNA nanotechnology, which uses DNA as a mimetic component in the construction of certain nanostructures. After indicating how this work can be understood in light of the proposed account of visual evidence, I will conclude the paper by discussing how realists and empiricists employ differently the notion of visual evidence. This leads to different appreciations of research done at the nanoscale.

\section{Visual Evidence Conceptualized}

In order to explore phenomena at the nanoscale, researchers need to construct, control and structure them via suitable instruments. Central among these instruments are probe microscopes, which make it possible to study the surface of various nanostructures. Scanning tunneling microscopes (STM) and atomic force microscopes (AFM) are two types of instruments that shape this research. These microscopes yield images that offer visual representations of corresponding features of the sample that cannot be detected otherwise except, of course, by using other instruments.

Nanoscale research, and, in particular, nanotechnology, is often characterized by the production of images that are supposed to offer evidence that certain relations hold among the nanoscale objects that are studied. It is by looking at these images that researchers assess how well they have managed to capture the relations in question. In typical cases, without seeing the images, without experiencing their content, researchers would not be in a position to know what they take to know about the sample. Thus, experiencing the microscopic images is a crucial component of the process. A description of the image would not have the same content as the image itself, any more than a description of a painting has the same content as the painting (Lehrer 2005; 2006). In order to know what a painting is like, in order to know the painting's content, it is crucial to see the painting itself. No matter how detailed a description of Goya's "Saturn Devouring one of His Sons" is, without actually seeing the painting, without experiencing it, one cannot know what the painting is like. Similarly, without actually seeing nanoscale images, without experiencing them, researchers cannot know what these images are like.

The content of images, whether in science or in art, has at least two components: what the image is about (the object-oriented component), and what the image is like (the experience-oriented component). Picasso's painting of Dora Maar is not only about Dora Maar, although Marr is certainly part of the content of the painting. It is also about what it is like to perceive Dora Maar from a cubist perspective, which is part of the experience involved in seeing the painting. Both features are central to the characterization of the painting's 
content. Similarly, the content of nanoscale images also has an object-oriented component and an experience-oriented component. For instance, an image of DNA molecules in a certain geometrical configuration is not only about DNA, although DNA is certainly part of the content of the image. It is also about what it is like to perceive DNA, structured in a certain way, from the perspective of an atomic force microscope, which is part of the experience involved in seeing the image. Both features are central to the characterization of the image's content.

So far, I have been describing the content of nanoscale images as though these images provided uncontroversial access to the way the items in the sample behave. Although researchers sometimes describe images in these terms, it is not clear that the phenomena require it. Perhaps we need not go as far as claiming that microscopic images are "public hallucinations" (see van Fraassen 2008, 101-11) analogous to rainbows, in that they can be seen by several observers and can even be captured in photographs (in this sense, they are public), but are not things (in this sense, they are hallucinations). The issue, however, is less whether these images are things, but what the content of these images amounts to. In particular, do nanoscale images provide evidence for what is happening in the sample or not? If so, what kind of evidence is it?

Visual evidence can be understood as the result of some partial mappings between the surface of the image and the sample, so that certain relations among the items in the sample are preserved and represented in the image (see Bueno 2006). The partial mappings are induced by the interaction between the microscope and the sample under study. For example, in the case of a scanning tunneling microscope (STM), the (conductive) tip of the microscope establishes a tunneling current with the (conductive) surface of the sample. By keeping the tunneling current constant and by scanning the surface of the sample with the STM, researchers produce images that provide information about the topography of the sample (see Binnig and Rohrer 1983; Chen 1993). If all goes well, there should be a partial match between the STM image and the corresponding features in the sample. But the correspondence is at best partial, given that not every aspect of the sample is selected for representation, due to the design of the instrument. For instance, features of the inner structure of the sample have no counterpart in an STM image. Similar points can be made about an atomic force microscope (AFM), except that in its case there is no tunneling current. Taking care to avoid damaging the sample's surface, researchers can study the surface even of non-conductive materials.

In several cases, in order to run experiments using STM or AFM, researchers design a theoretical image of what they take to be going on at the nanoscale. Such a theoretical image is often formulated as a schematic drawing (a schematic template) of some aspect of the surface of the sample. In these cases, the nanoscale image that is produced by the STM or the AFM (we can call it the empirical image) may be experienced as resembling the theoretical image that was used as a guide in the elaboration of the experiment. Given the resemblance between the theoretical and the empirical images, some researchers then infer 
that the corresponding configuration represented on the empirical image is found among the items in the sample.

Three components are then involved in cases of this sort: the theoretical image (the schematic template), the empirical image (the output of the relevant microscope), and the sample under study. Partial mappings between the theoretical image and the empirical image can be explicitly identified, given that researchers have direct access to both sorts of images. Given the partial mappings, researchers then infer that there is a corresponding match between the empirical image and the sample. But it is important to acknowledge that this is indeed an inference, given that no direct access to the items in the sample is available when one is dealing with nanoscale phenomena. In this way, due to the mappings in question, the empirical image can be taken as evidence that certain configurations among the items in the sample are present.

\section{Visual Evidence Applied}

As an illustration of this account of visual evidence, consider the case of DNA nanotechnology (Seeman 2003; 2005; Seeman and Belcher 2002). Biological nanostructures have a crucial property that chemists call molecular recognition - the fact that molecules of complementary types tend to attract each other. Due to this property, the self-assembly of certain nanostructures becomes possible. In particular, it is well known that DNA exemplifies two features of self-assembly: (a) the molecules involved have a strong affinity for each other (they clearly exemplify molecular recognition), and (b) the molecules form a predictable structure when they associate (which allows researchers to have some control over the outcome of the molecular interactions).

By carefully exploring these two features, researchers developed what is now called DNA nanotechnology. This approach allows them to build nanostructures from the bottom up, starting with chemical systems, and building more complex nanostructures along the way. More precisely, the approach uses DNA as a biomimetic component for self-assembly of non-biological structures by exploring the "sticky end" cohesion of DNA molecules. A sticky end is a short single-stranded overhang protruding from the end of a DNA molecule (Seeman 2003). Molecules with "sticky ends" are then tacked together.

By exploiting the self-assembling properties of DNA, Nadrian Seeman and his group at New York University have managed to construct two-dimensional arrays of DNA crystals in various shapes (see Ding et al. 2004). Their goal was to build complex nanostructures from DNA crystals in a triangular shape. With stable DNA triangles in place, the team aimed to combine six triangles to form a hexagonally shaped crystal. Dozens of such hexagons could then be, in turn, combined to form yet more complex honeycomb lattices. Throughout this process, a particular template guided the researchers: a theoretical image. This image schematically presented the DNA crystals in the various, progressively 
more complex, shapes (Ding et al. 2004, 10230, Figure 1). The team used an AFM to generate empirical images that provided visual evidence for the construction. In particular, honeycomb lattices formed by hexagons that, in turn, were composed by triangular crystals are all clearly represented (Ding et al. 2004, 10231, Figure 2). As Seeman and his group note: "The honeycomb structure of arrangements is evident from the [AFM] images" (Ding et al. 2004, 10230).

Clearly, in this case, there are partial mappings between the theoretical images (the schematic template) and the empirical images (the AFM micrographs). And, in terms of these mappings, the researchers concluded that the corresponding honeycomb lattices are also present in the sample. We have a clear case of visual evidence in action.

\section{Visual Evidence Problematized}

Does the formulation of visual evidence just discussed lend support to a realist interpretation of nanoscale images? That is, given the match between the theoretical and the empirical images, are we then entitled to conclude that the empirical images establish that the processes that are taking place in the sample behave in just the way that is represented in the images? I am not sure that we are. Realists can certainly adopt the account of visual evidence just offered, and disagree with this assessment. But in order to obtain a realist conclusion from the account of visual evidence, what realists will have to do is to interpret the account in fairly strong terms, that is, in terms of a definite match between the empirical image's structure and the corresponding features in the sample. But this interpretation is not forced by the account. It is also perfectly possible to interpret the account in more neutral terms.

As noted above, nanoscale images are the product of the interactions between the relevant microscopes and the sample. In many cases, the empirical images match fairly well the visual properties that were predicted via the schematic templates (the theoretical images), in the sense that there are partial mappings between such templates and the corresponding empirical images. The result is a perceived similarity between the two groups of images. The issue, however, is whether the shared structure between the empirical and theoretical images warrants the conclusion that the items in the sample have the same structure as well. Realists tend to claim that it does, and hence they take a stand on whether the empirical images provide visual evidence for the behavior of the nanoscale phenomena under study.

Empiricists, in turn, tend to be more skeptical. They note that it is assumed that nanoscale empirical images yield information about the behaviour of the components in the sample. These images do provide observable traits that need to be explained. As Bas van Fraassen notes, various microscopes can be thought of as "engines of creation", engendering new observable phenomena that needs to be accounted for (van Fraassen 2008, 96-101). But the realist still needs to infer that the best explanation as to why the empirical images have the configurations they have on their surfaces is because there are genuine 
nanoscale phenomena in the sample that cause these configurations. Empiricists will challenge, of course, whether this is indeed the best account of the resulting images, given the well-known concerns about inference to the best explanation (see, e.g., van Fraassen 1989).

But even if we leave these concerns aside, another concern emerges about the connection between the empirical image and the sample. Even if there are partial mappings between the sample and the empirical image, given that we have no direct access to the sample, we cannot know exactly the shape of these mappings. That is why some kind of link from the way the empirical images look to the way one expects the sample to behave is unavoidable. And here we experience a gap.

When reports of the empirical images are given, a delicate ambiguity is in place. As Seeman and his team note: "The honeycomb structure of arrangements is evident from the [AFM] images" (Ding et al. 2004, 10230). When we look at the empirical AFM images, the honeycomb structure of arrangements is indeed evident. In fact, we can see honeycomb structures in the AFM images. As a description of the images, this is literally correct. The ambiguity arises when we interpret "honeycomb structure" to stand not for a description of the images, but for a description of the sample. There is an assumption of transparency in place here, as though simply by looking at the AFM images we could see exactly what the sample looks like. An analogy would be to look at a photograph of Quine to see how Quine looks. But AFM images, created as the outcome of the interaction of the tip of the microscope and the surface of the sample, cannot be transparent in this way (assuming, for the sake of argument, that photographs are indeed transparent). After all, an AFM image offers something analogous to what a blind person would "see" in the surface of an object by systematically touching it. The degree of perturbation to the system in the case of AFM, as in the case of a person feeling an object with his or her hand, is greater in both cases than what would happen if the person is perceiving low-energy light being reflected from that surface. It is difficult to make sense of transparency in these cases. It is likewise difficult to make sense of the idea that the honeycomb structure evident in the AFM images corresponds exactly to the way things are in the sample. Some link from the empirical images to the sample is still needed.

\section{Conclusion}

A tentative account of visual evidence at the nanoscale was offered. In principle, both realists and empiricists can adopt the account. Realists will typically invoke a particularly strong interpretation of the account, inferring that the items in the sample behave in the way represented in the empirical images. Empiricists, in turn, will typically note that the evidence is less clear-cut and far less conclusive than realists may taken it to be. Visual evidence may not always be exactly what meets the eye. 
OTÁVIO BUENO

Department of Philosophy

University of Miami

Coral Gables, FL 33124-4670,

U.S.A.

otaviobueno@mac.com

\section{References}

Binnig, G., and H. Rohrer. 1983. Scanning Tunneling Microscopy. Surface Science 126: 236-44.

Bueno, O. 2006. Representation at the Nanoscale. Philosophy of Science 73: 61728.

Chen, C. J. 1993. Introduction to Scanning Tunneling Microscopy. New York: Oxford University Press.

Ding, B., R. Sha, and N. Seeman. 2004. Pseudohexagonal 2D DNA Crystals from Double Crossover Cohesion. Journal of the American Chemical Society 126: 10230-31.

Lehrer, K. 2005. Representation in Painting and Consciousness. Philosophical Studies 117: 1-14.

Lehrer, K. 2006. Knowing Content in the Visual Arts. In Knowing Art: Essays in Aesthetics and Epistemology, eds. M. Kieran and D. Lopes, 1-18. Dordrecht: Springer.

Seeman, N. 2003. DNA in a Material World. Nature 421: 427-431.

Seeman, N. 2005. From Genes to Machines: DNA Nanomechanical Devices. Trends in Biochemical Sciences 30: 119-25.

Seeman, N., and A. Belcher. 2002. Emulating Biology: Building Nanostructures from the Bottom Up. Proceedings of the National Academy of Science 99: 6451-55.

van Fraassen, B. 1989. Laws and Symmetry. Oxford: Clarendon Press.

van Fraassen, B. 2008. Scientific Representation: Paradoxes of Perspective. Oxford: Clarendon Press. 